\title{
UTILISATION DE LA MÉTHODE DES CAPTURES SUCCESSIVES (DE LURY) POUR L'ÉVALUATION DES PEUPLEMENTS PISCICOLES
}

\author{
M. I.ATRIENT et I. I.AMIARQIT: \\ Staliun d'Hydrobinlagie, I. N.R.A., \\ B. $I^{\prime} .79,61200$ Biarm:
}

\begin{abstract}
RÉSUMÉ
I.a méthode des captures successives (DE LCRY) a été utilisée pour l'évaluation de trois types de peuplements piscicoles. Les résultats obtenus avec deux pèches et trois pêches sont comparés entre eux : la méthode des captures-recaptures (PETERSEx) a également été comparée avec la méthode DE LuRY deux pêches.

D'une manière générale, il ressort de ce travail que la méthode Petersfox doit être utilisée dans le cas de faibles efficacités et de peuplements peu importants. Ftant donné les conditions statistiques draconiennes devant être réalisées lors de l'emploi de la méthode des captures successives (I) I,URY), il semble préférable de réserver son emploi au cas de fortes efficacités ou de peuplements importants.

On propose l'emploi d'une stratégie, basée sur l'importance relative des captures, pour l'exécution des inventaires piscicoles par la méthode DE LURY.
\end{abstract}

Mots clés : évaluation de peuplements, mathématiques

\section{I. - INTRODUCTION}

La méthode d'inventaire DE L,URY est utilisée depuis un certain temps à l'étranger (DE LURY, I947). La méthode consiste à effectuer un certain nombre de pêches successives. Les poissons capturés à chaque pêche ne sont ni marqués, ni remis à l'eau.

Les hypothèses de base pour l'emploi de la méthode sont les suivantes : effort de pêche constant et absence de déplacements des poissons en dehors du secteur de capture. İ̀n toute rigueur, les résultats obtenus ne s'appiqquent qu'à la portion de rivière inventoriée; en fait, on admet (CUINat et Dumas, sous presse) la possibilité d'extrapoler les résultats d un secteur représentatif à l'ensemble d'une portion de rivière. On appelle efficacité d'un engin de pêche, dans un milieu donné, le rapport entre le 
nombre de poissons capturés et le nombre de poissons existant dans le milieu; cette efficacité dépend donc évidemment à la fois du milieu et de l'effort de pêche.

Pour un même milieu, elle ne dépend que de l'effort de péche et il revient au mème dans ce cas de dire " effort de pêche constant "ou " efficacité constante".

DAGET (I97I) a donné un exemple d'application de la méthode avec Io pêches successives. Cunat et Itras (sous presse) ont donné le mode de calcul du peuplement le plus probable et des limites de confiance dans le cas de'deux pêches.

Depuis quelques années cette méthode est également utilisée en France. Sa facilité d'exécution, par rapport à la méthode Petersex, lui vaut les faveurs des utilisateurs, en particulier des Chefs de Régions piscicoles du Conseil supérieur de la Pêche. Devant les difficultés rencontrées par ceux-ci dans l'application de la méthode et l'interprétation des résultats, il a semblé utile de préciser les conditions d'application de la méthode De L,erY, d'indiquer l'exécution détaillée des calculs dans le cas des deux pêches et trois pêches et enfin, par des exemples précis, de comparer les résultats obtenus avec cleux pêches et trois pêches avec la méthode PETERSEx.

\section{II. - CONDITIONS D'APPLICATION DE LA MÉTHODE}

Pour évaluer le peuplement le plus probable, on trace alors la droite de régression, avec $y_{i}^{\prime}=$ nombre de poissons capturés à la pêche $i$,

$$
y_{i}=f\left(x_{i}=\sum_{j=1}^{j=i-1} y_{j}\right)
$$

Dans le cas de 2 pêches seulement, on joint les points $0, y_{1}$, et $y_{1}, y_{2}$. Le peuplement le plus probable est donné par l'abscisse de l'intersection de cette droite et de l'axe des $x$. Les limites de confiance sont données dans le cas de 2 pêches, par la formule de SEBER et LE CREN (1967); dans le cas de plus de 2 pêches elles sont données par les points obtenus sur l'axe des $x$ en tenant compte des limites de confiance du coefficient angulaire de la droite de régression. On doit remarquer que l'efficacité devant être constante, la méthode ne s'applique en toute rigueur qu'à des groupes de tailles pour lesquels l'efficacité de l'engin de pêche est approximativement constante. En fait, il est possible (DAGET, I97I), en première approximation, d'admettre une efficacité moyenne globale pour l'ensemble des tailles et dans le cas de 3 pêches et plus d'utiliser les poids de poissons capturés pour évaluer le stock pondéral.

\section{III. - CALCUL DES PEUPLEMENTS les PIUS PROBABLES \\ ET DES LIMITES DE CONFIANCF,}

\section{Cas de 2 pêches}

Soit $c_{1}$ et $c_{2}$ le nombre de poissons capturés en $I^{\text {re }}$ et $2^{\mathrm{e}}$ pêche. On vérifie (SEBLR et LE (REN, I967) :

Io que

$$
c_{1}>c_{2}
$$


2" que

$$
\frac{c_{(2}^{2}\left(c_{1}-c_{2}\right)^{2}}{c_{2}^{2}\left(c_{1}+c_{2}\right)}>\mathrm{I} 6
$$

Si ces deux conditions ne sont pas vérifiées, il n'est pas possible d'évaluer le peuplenent le plus probable ni a fortiori les limites de confiance. On peut simplement dire que $\mathrm{P}^{\prime}>c_{1}+c_{2}$

Si cette condition est remplie, on a le peuplenent le plus probable $\mathrm{P}$, abicisse de l'intersection de la droite avec l'axe des $x$ :

Soit :

$$
y-c_{1}=\frac{c_{2}-c_{1}}{x} c_{1}
$$

$$
\mathrm{P}=c_{1} \cdots c_{2}^{2}
$$

La variance de $P$, calculée par la méthode du maximum de vraisemblance, s'écrit :

$$
\operatorname{var} \mathrm{P}=\frac{c_{1}^{2} c_{2}^{2}\left(c_{1}+c_{2}\right)}{\left(c_{1}^{-}-c_{2}\right)^{2}}
$$

d'oì l'on tire les limites de confiance de $P$, avec $9.5 \mathrm{p}$. Ioo de sécurité:

$$
\mathrm{P}-2 \sqrt{\operatorname{var} \overrightarrow{\mathrm{P}}}<\mathrm{P}<\mathrm{P}+2 \sqrt{\text { var } \overrightarrow{\mathrm{P}}}
$$

11 arrive que $\mathrm{P}-2 \sqrt{\text { var } \mathrm{P}}$ soit inférieur à $c_{1}+c_{2}$; on prencl alors comme limite inférieure la quantité $c_{1}+c_{2}$.

\section{Cas de 3 pêches}

Soit $c_{1}, c_{2}, c_{3}$ les captures en $\mathrm{I}^{\mathrm{re}}, 2^{\mathrm{e}}$ et $3^{\mathrm{e}}$ pêches. On vérifie que $c_{1}>c_{2}>c_{3}$. In effet, s'il est possible théoriquement de construire une droite de régression ct estimer le peuplement et les limites de confiance, avec par exemple $c_{2}>c_{1}>c_{3}$, dans la pratique si l'on obtient $c_{2}>c_{1}$ ou $c_{3}>c_{3}$, on en déduit que l'efficacité de l'engin de pêche est très mauvaise et que la méthode De LURY est alors difficilement applicable, comme on le verra plus loin.

Si cette condition n'est pas remplie, on peut simplement dire que :

$$
\mathrm{P} \geqslant c_{1}+c_{2}+c_{3}
$$

La droite de régression s'écrit :

$$
y-\bar{y}=b(x-\bar{x}),
$$

la valeur absolue de $b$ pouvant être considérée comme l'efficacité moyenne de l'ensemble des pêches.

$$
b=\frac{\mathrm{I}}{\sum_{i=1}^{i=n}\left(x_{i}-\bar{x}\right)^{2}} \times \sum_{i=1}^{i-n}\left(x_{i}-\bar{x}\right) y_{i}=\frac{\left(x_{1}-\bar{x}\right) y_{1}+\left(x_{2}-\bar{x}\right) y_{2}+\left(x_{3}-\bar{x}\right) y_{3}}{\left(x_{1}-\bar{x}\right)^{2}+\left(x_{2}-\bar{x}\right)^{2}+\left(x_{3}-\bar{x}\right)^{2}}
$$

$n$ étant le nombre de pêches. 


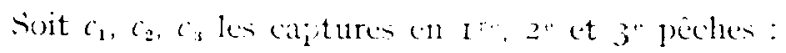

$$
\begin{aligned}
& x_{1}, 0 \quad x_{2}=c_{1} \quad x_{3} \quad \therefore c_{1} \cdots c \\
& r_{1}-c_{1} \quad y_{2}-c_{2} \quad y_{3}-c_{3} \\
& \bar{x}=2 c_{1}-c_{2} \quad x_{1} \quad \bar{x}=2 c_{1}-c_{2} \quad x_{2}-\bar{x} \quad c_{1}-c_{2} \\
& x_{3}-\bar{x}=-2 c_{2} \cdots \cdot c_{1} \\
& \overline{1}-=c_{1} \div c_{2}+c_{3}
\end{aligned}
$$

d'où

$$
1,-\mathrm{I}_{2} c_{1}\left(c_{1}-c_{3}\right)-c_{2}\left(c_{2}-c_{3}\right)+c_{1}^{2}-c_{2} c_{3}
$$

On doit vérifier ici :

$I^{\circ}$ que $b$ est négatif (ce qui est certain si $c_{1}>c_{2}>c_{3}$ );

2" que le coefficient de corrélation

$$
r=\sum_{i=1}^{\sum_{i=1}^{i=n}\left(x_{i}-\bar{x}\right)\left(y_{i}-\bar{y}\right)}
$$

c.t supéricur, pour 3 péches, à 0,097 (corrélation différente de 0 à 95 p. Ioo de seccurité).

Si cette condition n'est pas remplie, l'écart-type de $b$ est si grand que la limite supérieure de la population est négative, c'est-à-dire sans signification pratique, et que la limite inférieure est très inférieure aux captures cumulées; la valeur la plus probatsle n'a guère plus de signification que la quantité $c_{1}+c_{2}+c_{3}$. On peut seulement dire alors que $\mathbf{P} \geqslant c_{1}+c_{2}+c_{3}$.

L'abscisse de l'intersection de la droite de régression avec l'axe des $x$ s'écrit :

$$
\mathrm{P}=\bar{x}-\frac{\bar{y}}{b}
$$

b) étant toujours négatif, et $|b|$ sa valeur absolue :

$$
\mathbf{P}: \bar{x} \div \bar{y}
$$

Calcul des limites de confuanci.

On doit d'abord calculer la variance de $b$.

La variance de $b$ s'écrit (DAGNILIE, I970) :

$$
\operatorname{var} b=\left[\Sigma\left(y_{i}-\bar{y}\right)^{2}-\left[\left(x_{i}-\bar{x}\right)\left(y_{i}-\bar{y}\right)\right]^{2}\right] \times(n-\bar{x}) \frac{\mathrm{I}}{\Sigma\left(x_{i}-\bar{x}\right)^{2}}
$$


soit ici :

$$
\text { var } b=\frac{c_{1}^{2}+c_{2}^{2}+c_{3}^{2}-\left(c_{1} c_{2}+c_{2} c_{3}+c_{1} c_{3}\right)-b^{2}}{c_{1}^{2}+c_{2}^{2}+c_{1} c_{2}}
$$

$b$ étant donné par (I).

In fait, le calcul de $b$ ct de la variance de $b$ présenté ici n'est valable qu'en première approximation. Iìn effet, les captures à charque pêche ne sont pas connues arec la même précision, puisque leur nombre diminue régulièrement. I,e calcul de $b$, et de la variance de $b$ est plus correct en pondérant les points de la droite de régression par l'inverse des effectifs restant dans le milieu à la pêche $i:$ I soit : P I $\mathrm{P}$ étant estimé par $\mathrm{P}=\bar{x}+\frac{\bar{y}}{|\bar{b}|}$ on obtiendra ainsi une nouvelle valeur $\mathrm{P}^{\prime} \mathrm{de}$ $\mathrm{P}\left(\mathrm{P}^{\prime}=\bar{x}+\frac{\bar{y}}{|\bar{b}|}\right)$. Cette manière de procéder a l'avantage de donner un poids statistique plus fort aux points relatifs aux dernières captures, ce qui est raisonnable dans le cas d'une forte efficacité. Cependant, étant donné la complexité du calcul, on a préféré s'en tenir dans la suite du travail à l'estimation de $b$ et le la variance de $b$ telles qu'elles sont présentées.

On tire de $b$ et var $b$ les limites de confiance de $b$, pour $n-2=\mathrm{I}$ degré de liberté et $95 \mathrm{p}$. Ioo de sécurité, avec $t 0,975=\mathrm{I} 2,73$.

$$
b-\mathrm{I} 2,73 \sqrt{\operatorname{var} b}<b<b+\mathrm{I} 2,73 \sqrt{\operatorname{var} b}
$$

et les limites de confiance de $\mathrm{P}$ :

Limite inférieure: $\quad \mathrm{P}_{1}=\bar{x}+\frac{\bar{y}}{\mid b-\mathbf{1} 2,73 \sqrt{\operatorname{var} b \mid}}$

Limite supérieure : $\quad \mathrm{P}_{2}=\bar{x}+\frac{\bar{y}}{\mid b+12,73 \sqrt{\operatorname{var} b \mid}}$

$P_{1} \leqslant P \leqslant P_{2}$.

Graphiquement les estimations de $\mathrm{P}$ et de l'intervalle de confiance sont présentées sur la figure ci-dessous, dans le cas de 3 pêches.

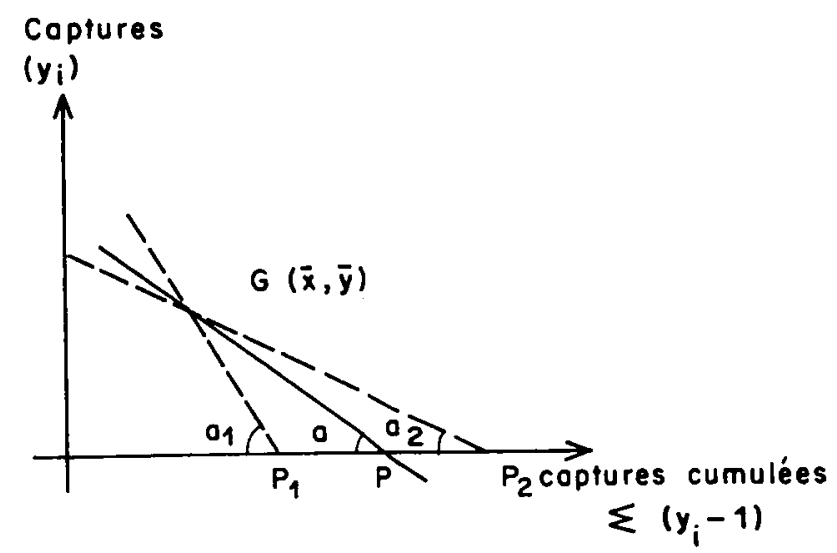

avec $b==\operatorname{tg} a, b-\mathrm{I} 2,73 \sqrt{\operatorname{var}} \vec{b}=\operatorname{tg} a_{1}$ et $b \div 12,73 \sqrt{\operatorname{var} b}=\operatorname{tg} a_{2}$ 
Remarques: Si la limite $\mathrm{P}_{1}$ est inférieure à la somme de captures, on prend cette dernière comme limite inférieure.

Si $b+\mathbf{1} 2,73$ var $b>0$, la limite supérieure est sans signification.

Les limites de confiance sont dissymétriques par rapport au peuplement le plus probable, la limite inférieure en étant plus proche que la limite supérieure. Cette dissymétrie est générale dans l'estimation de limites de confiance d'une grandeur à partir de la détermination des limites de confiance d'une autre grandeur, inverse de la première.

\section{IV. - EXEMPLES D'APPLICATION}

Exemple 1. Évaluation de peuplements d'anguilles et de varrons capturés dans le Couesnon et le Nançon (rivières bretonnes). Les données sont tirées de BEAUDours et Pontini (1973). On calculera le peuplement et ses limites de confiance par la méthode De LuRY trois pêches, puis par la méthode De LuRY deux pêches en utilisant seulement les deux premières pêches; on pourra ainsi comparer les résultats. Les données et résultats sont rassemblés dans le tableau $\mathrm{I}$.

Le tableau $\mathrm{I}$ montre que pour les anguilles du Nançon et les deux peuplements de vairons étudiés, il n'est pas possible, par l'une ou l'autre méthode, d'évaluer le peuplement le plus probable ni ses limites de confiance (respectivement $r<0,997$ et conditions SEBER et LE CREN non satisfaites).

Pour les anguilles du Couesnon on obtient, pour le peuplement le plus probable, avec 3 pêches 46 et avec 2 pêches 44 .

Le rapport too $\frac{\mathrm{P}_{2}-\mathbf{P}_{1}}{{ }_{\mathbf{P}}}$, qui exprime l'importance relative de l'intervalle des limites de confiance vis-à-vis du peuplement le plus probable, donne une indication sur la précision de la méthode employée : plus il est faible, meilleure est la précision. Ici, il est de $39 \mathrm{p}$. Ioo dans le cas de 2 pêches et de $\mathrm{I} 3 \mathrm{p}$. Ioo dans le cas de 3 pêches.

Le fait d'effectuer une $3^{\text {e }}$ pêche permet donc :

Io d'améliorer la détermination du peuplement le plus probable; on peut penser en effet, si on admet (TImmermans, 1957) que l'efficacité.baisse à chaque pêche, que les peuplements les plus probables sont sous-estimés;

$2^{\circ}$ d'améliorer la précision de l'inventaire en resserrant nettement les limites de confiance du peuplement.

Exemple 2. Il s'agit d'évaluer le stock pondéral de peuplements de Tilapia nilotica L. et Tilapia $3 / 4$ (hybride à position systématique incertaine), issus de 2 secteurs différents du I ac Itasy à Madagascar. Dans ce cas, la méthode Ie LURY deux pêches ne peut être employée, car la vérification de la condition de SEBBER et LE CREN doit être effectuée à l'aide des nombre de poissons. La méthode De I,URY trois pêches peut être utilisée car les calculs nécessaires à l'établissement de la droite de régression peuvent être réalisés à partir de données pondérales.

Le tableau 2 rassemble les données et les résultats. Il montre que l'intervalle des limites de confiance est étendu et donc que le stock n'est pas connu avec précision. 


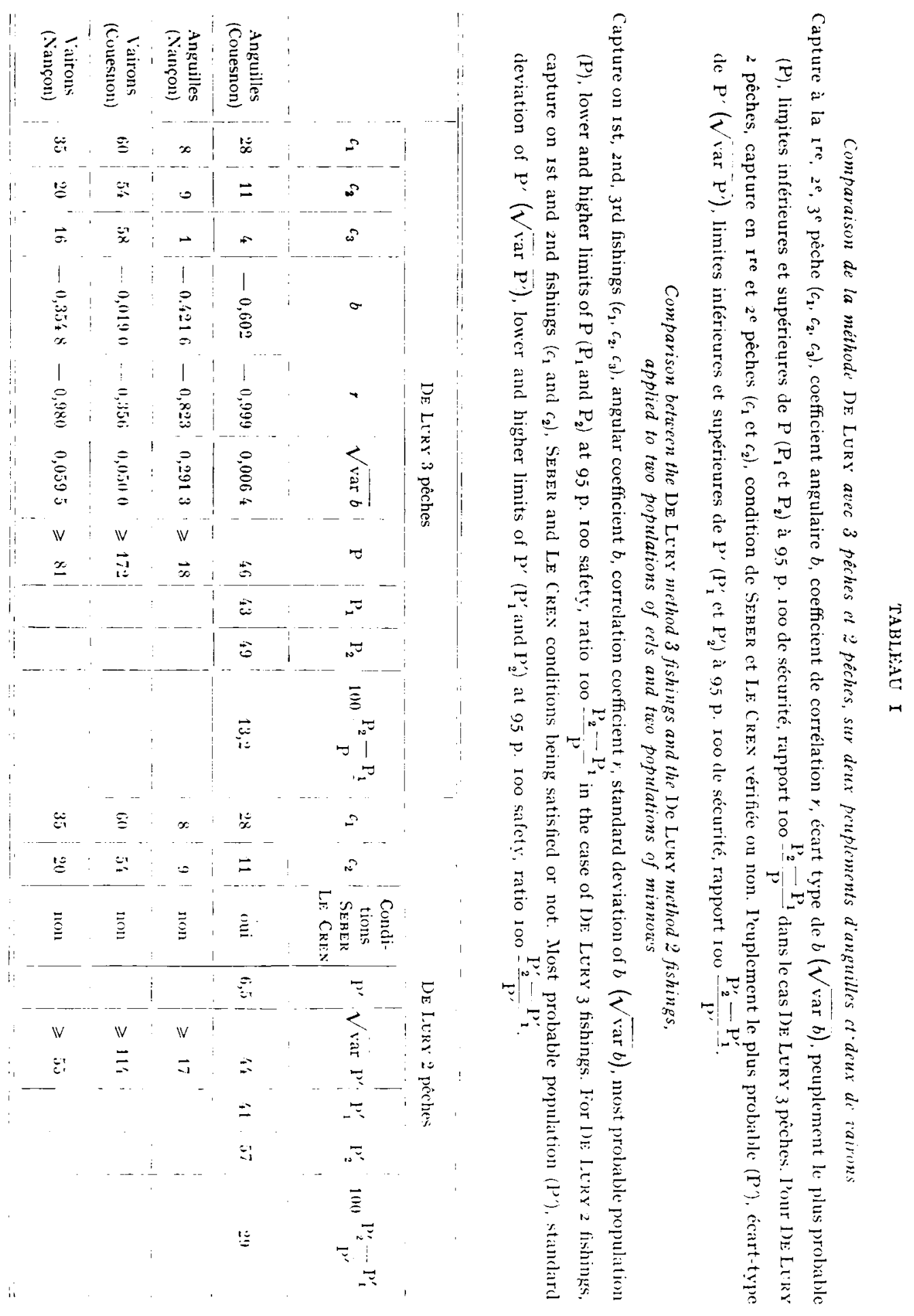


TABLEAU 2

Méthode DE Lury 3 pêches appliquée à quatre populations de Tilapia

Captures en $\mathbf{k g}$ en $\mathbf{I}^{\mathrm{re}}, 2^{\mathbf{e}}$ et $3^{\mathrm{e}}$ pêches $\left(c_{1}, c_{2}, c_{3}\right)$ coefficient de corrélation $(r)$, coefficient angulaire $(b)$, écart-type de $b(\sqrt{\operatorname{var} b})$, peuplements les plus probables ( $\mathrm{P}$ en $\mathrm{kg}$ ), limites inféricures $\left(P_{1}\right)$ et supérieures $\left(\mathrm{P}_{2}\right)$ en $\mathrm{kg}$, pour 2 peuplements de Tilapia nilotica et 2 peuplements de Tilapia $3 / 4$.

DE LURY method 3 fishings applicd to 4 populations of Tilapia

Captures in $\mathrm{kg}$ on $\mathrm{Ist}$, and and 3 rd fishings $\left(c_{1}, c_{2}, c_{3}\right)$, correlation cocfficient $r$, angular coefficient $b$, standard deviation of $b(\sqrt{\operatorname{var} b})$, most probable populations ( $\mathrm{P}$ in $\mathrm{kg}$ ), lower limits $\left(\mathrm{P}_{1}\right)$ and higher limits $\left(\mathrm{P}_{2}\right)$ in $\mathrm{kg}$, for 2 populations of Tilapia nilotica and 2 populations of Tilapia $3 / 4$.

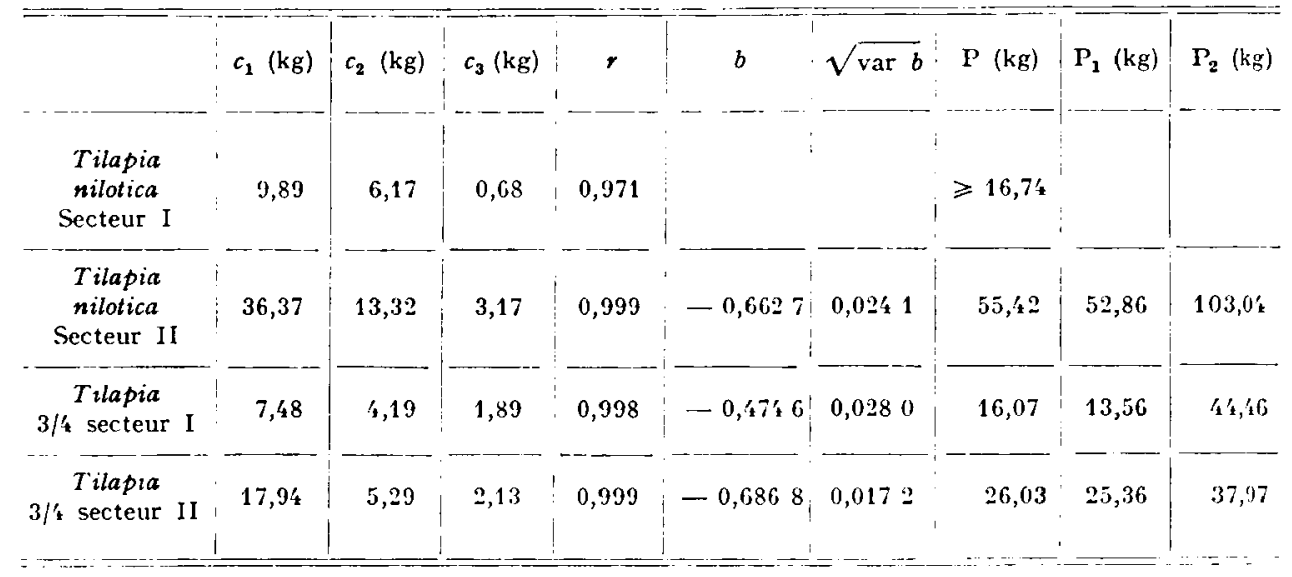

Exemple 3. Cet exemple est tiré d'un inventaire de population de truites, effectué sur la cressonnière d'Agos (CunNat et Dumas, sous presse) par la méthode Petersen. A l'aide de ces données on peut "fabriquer" des données correspondant à la méthode DE LuRy deux pêches, de la façon suivante :

- le nombre de poissons non marqués capturés en Ire pêche PETERSF constitue le nombre de poissons capturés en $\mathrm{I}^{\mathrm{re}}$ pêche De LURY $\left(c_{1}\right)$.

- le nombre de poissons non marqués capturés en $2^{\mathrm{e}}$ pêche Pertarsis constitue le nombre de poissons capturés en $2^{e}$ pêche De LURY $\left(c_{2}\right)$.

L es poissons sont ici rassemblés en quatre grands groupes de taille; à l'intérieur de chacun des groupes l'efficacité peut être considérée comme constante.

Le tableau 3 rassemble les résultats calculés suivant les 2 méthodes (I)E: LuRY et Petersens). Ce tableau montre que les deux méthodes sont équivalentes lorsqu'on a affaire à de fortes efficacités (groupe III et IV, poissons de grandes tailles).

Lorsque l'efficacité est moyenne (groupe II) la méthode De LuRY sous-estime

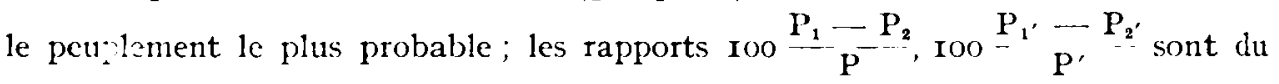
même ordre de grandeur. Lorsque l'efficacité est faible (groupe r), la méthode DE LURY sous-estime le peuplement et donne un rapport $\frac{P_{1}-P_{2}}{P}$ environ 2 fois supérieur à celui obtenu par la méthode Petrasfr. Ce résultat est logique car la 


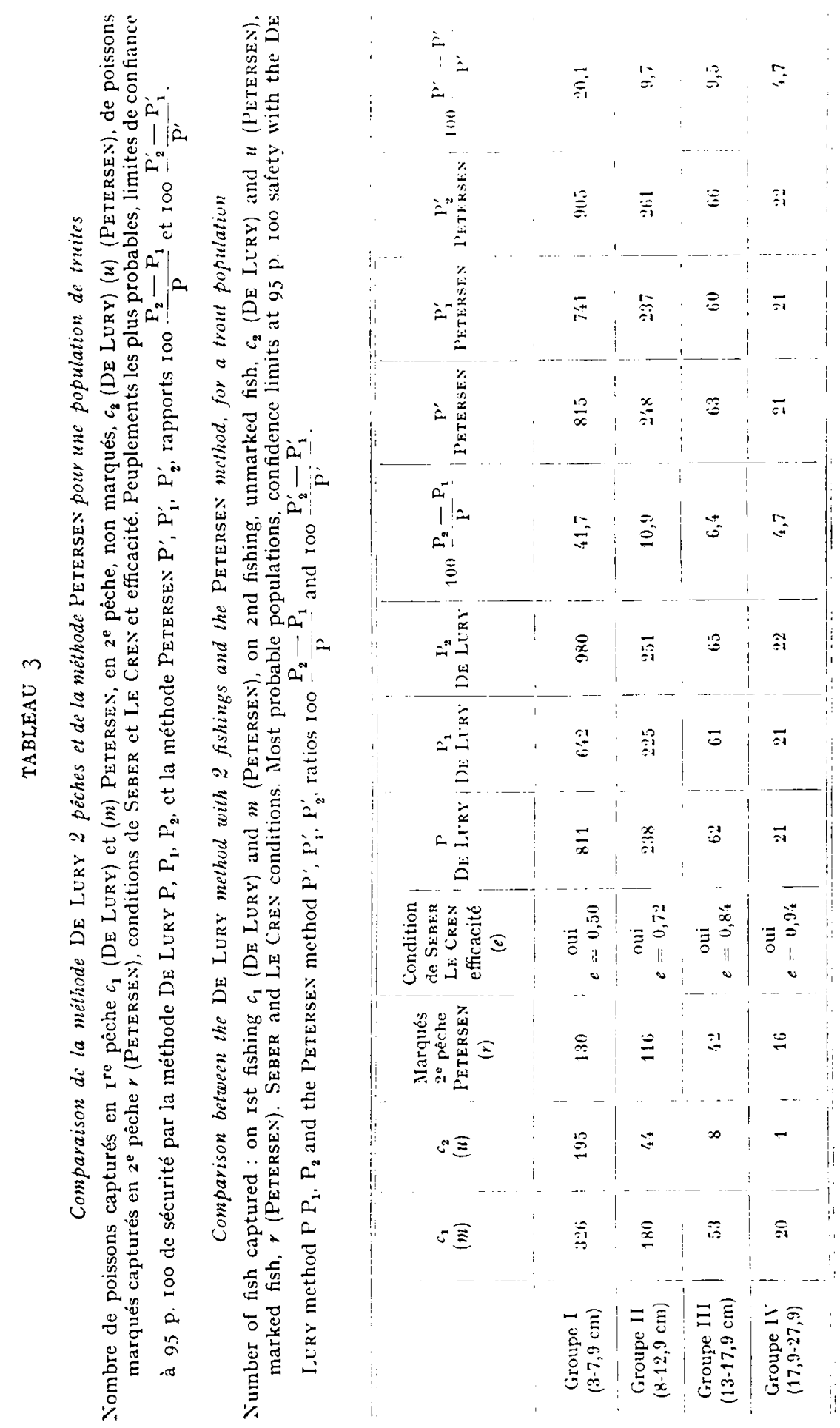


méthode de Petersex apporte, avec le nombre de poissons non marqués capturés en deuxième pêche, une information supplémentaire par rapport à la méthode DE LURY; ceci n'est valable qu'en admettant une hypothèse de base de la méthode PETERSEx : l'égale probabilité de capture des marqués et non marqués.

\section{V. - CONCLUSIONS}

De l'examen de ces 3 exemples ressort nettement la difficulté d'emploi de la méthode DÉ LuRY à l'aide de 2 pêches et de 3 pêches, compte tenu des conditions statistiques draconiennes à respecter (condition de SEBER et LE CREN pour 2 pêches, valeur limite élevée du coefficient de corrélation $(0,997)$ et du coefficient multiplicateur de 1'écart-type $t 0,975=$ I2,7I pour 3 pêches). En outre, même quand la méthode peut s'appliquer, elle a tendance à sous-estimer le peuplement. Dans la plupart des cas, il est donc préférable d'utiliser la méthode PETERSE., sous peine de ne pouvoir tirer aucune conclusion de l'inventaire, quant à l'évaluation du peuplement le plus probable et de ses limites de confiance. Lorsqu'on tient à utiliser la méthode DE L,URY il semble indispensable d'augmenter le nombre de pêches car dans ce cas les conditions statistiques sont moins draconiennes (pour 4 pêches : $t 0,975=4,303$ et $r=0,95$; pour 5 pêches : $t 0,975=3,182$ et $r=0,88$, etc.)

Sur le plan pratique, on peut proposer la stratégie suivante : on effectue les $I^{\text {re }}$ et $2^{\mathrm{e}}$ pêches; sur 1'ensemble des poissons on vérifie sur le terrain la condition de SEBER et LE CREN :

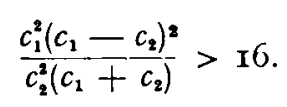

Si elle est vérifiée, on peut s'arrêter là ; sinon on effectue une $3^{e}$ pêche et on calcule $r$ à l'aide de la formule suivante :

$$
r=\frac{\sum_{i=1}^{i=n}\left(x_{1}-\bar{x}\right)\left(y_{1}-\bar{y}\right)}{\sqrt{\sum_{i=1}^{1=n}\left(x_{1}-\bar{x}\right)^{2} \quad \sum_{i=1}^{1-n}\left(y_{1}-\bar{y}\right)^{2}}}
$$

Si $r>0,997$ on s'arrête là, sinon on effectue une $4^{\mathrm{e}}$ pêche et on vérifie si $r>0,95$ et l'on continue ainsi jusqu'à ce que l'on ait un coefficient de corrélation supérieur à la valeur limite.

La valeur limite de $r$ en fonction du nombre de pêches est donnée ici :

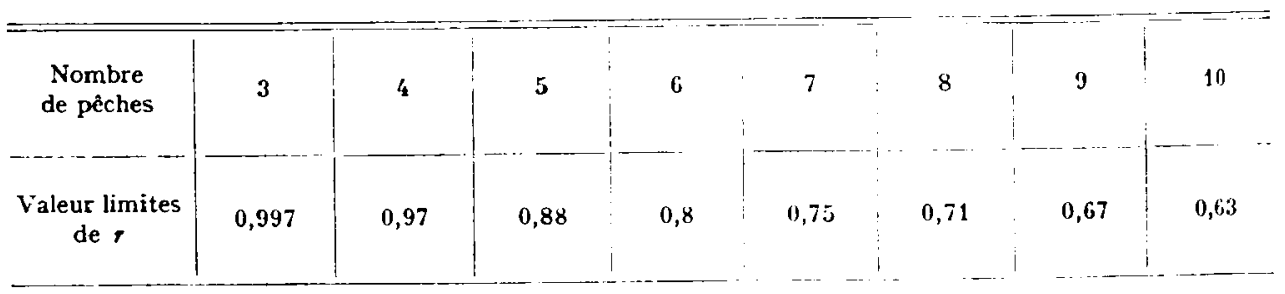

(Table tirée de LAmotte, 1957) 
Il est certain que l'exécution des calculs de $r$ sur le terrain au cours des opérations de pêche n'est pas matériellement facile.

$\mathrm{Si}$ on n'a pas la possibilité d'effectuer le calcul, on peut appliquer les règles suivantes pour 3 pêches, qui sont arbitraires et approximatives parce que simples, mais qui permettront d'avoir des résultats significatifs:

I" Moins de 10 poissons en $1^{\mathrm{re}}$ pêche.

La population ou l'efficacité sont très faibles. On ne peut rien évaluer avec certitude.

$2^{\circ}$ Entre 11 et 50 poissons en $1^{\text {re }}$ pêche.

On ne doit pas capturer en $2^{\mathrm{e}}$ pêche plus des $\frac{2}{3}$ du nombre de poissons pris en I $^{\text {re }}$ pêche; la même condition s'applique à la $3^{\mathrm{e}}$ pêche, soit :

$$
c_{2}=\frac{2}{3} c_{1} \quad \text { et } \quad c_{3}=\frac{2}{3} c_{2}
$$

Exemple avec $\quad c_{1}=45 ; \quad c_{2}=29 ; \quad c_{3}=\mathrm{I} 8$.

On obtient pour $e$ (efficacité moyenne de l'ensemble cles pêches) $e=0,364$; $r=0,9998 ; \quad \mathrm{P}=\mathrm{I} 24 ; \quad \mathrm{P}_{1}=\mathrm{I09} ; \quad \mathrm{P}_{2}=149$.

$3^{\circ}$ Entre 50 et 100 poissons en $1^{\mathrm{re}}$ pêche.

On ne doit pas capturer en $2^{\mathrm{c}}$ péche plus des $\frac{3}{4}$ du nombre de poissons pris en $I^{\text {re }}$ pêche; la même condition s'applique à la $3^{\mathrm{e}}$ pêche, soit :

$$
c_{2} \leqslant \frac{3}{4} c_{1} \quad \text { et } \quad c_{3} \leqslant \frac{3}{4} c_{2}
$$

Exemple avec $\quad c_{1}=80 ; \quad c_{2}=60 ; \quad c_{3}=42$.

On obtient $e=0,270 ; \quad r=0,99^{8} ; \quad \mathrm{P}=298 ; \quad \mathrm{P}_{1}=208 ; \quad \mathrm{P}_{2}=733$.

$4^{\circ}$ Au-delà de 100 poissons en $1^{\text {re }}$ pêche.

On ne doit pas capturer en $2^{\mathrm{e}}$ pêche plus de $90 \mathrm{p}$. Ioo du nombre des poissons pris en $\mathrm{I}^{\text {re }}$ pêche; la même condition s'applique à la $3^{\mathbf{e}}$ pêche.

Exemple avec $\quad c_{1}=200 ; c_{2}=180 ; c_{3}=160$.

On obtient $c=0, \mathrm{I0}_{5} ; \quad r=0,9995 ; \quad \mathrm{P}=\mathrm{I} 906 ; \quad \mathrm{P}_{1}=\mathrm{I} 429 ; \quad \mathrm{P}_{2}=2980$

On voit sur ces 3 exemples que plus le stock est important, moins l'efficacité limite pour permettre l'évaluation du stock doit être élevée; il reste cependant que plus les efficacités sont fortes, plus l'intervalle des limites de confiance est resserré.

Remarque : L'exécution manuelle de l'ensemble des calculs nécessaires pour une diagnose démographique est fastidieuse pour les méthodes PETERSEN et DE LURY deux pêches, et très longue à partir de DE LuRY trois pêches. C'est pourquoi des programmes de calcul des éléments nécessaires au biologiste des pêches, à partir des données brutes de terrain, sont en cours de réalisation. Ces programmes pourront être utilisés quelle que soit la méthode employée (PETERSEN ou DE LURY). 


\title{
SUMMARY
}

\author{
UTIIIZATION OF THE DE LURY METHOD BY SUCCESSIVE CAPTURES \\ FOR THE ESTIMATION OF FISH STOCKS
}

The DF L, TKY method by successive captures has been used for the estimation of three types of fish stocks. The results obtained with two and three fishing runs are compiered ; the PETERSEN method by capture-recapture has also been compared with the two fishing runs method.

On the whole, this study shows that the PETERSEN method should be used with low efficiencies and little stocks. According to the strict statistical conditions to be worked out with the DE LURY method. it seems advisable to use it for high efficiencies or large stucks.

A way of proceeding is proposed, which is based on the relative importance of captures, for working out the fish inventories with the DE LURY method.

\section{RÉFÉRENCES BIBLIOGRAPHIQUES}

Beavdoutx P., Pontini G., 1973. Étude hydrobiologique du bassin du Couesnon. Diplòme d'Agronomie approfondie, E. N. S. A., Rennes, 46 p. ronéotypées.

Clinat R., Dumas J. Diagnose écologique en cours d'eau à Salmonidés. Méthode et exemple. E. a.I.F. A.C., Tech. Pp. (sous presse).

DAGET J., 197I. L'échantillonnage des peuplements de poissons d'eau douce. In LAMotTe et BockLIÉRE. Problèmes d'écologie : l'échantillonnage des peuplements animaux des milieax aquatiques. I vol., I 94 p., Masson, Paris.

DAgnelie P., 1970 . Theorie et méthode statistiques. Tome 2, 45 I p., Duculot, Gembloux.

DE LURY D. B., 1947. On the estimation of biological populations. Biometrics, 3 (4), 145-167.

LA MOTтE M., 1957. Initiation aux méthodes statistiques en biologie. I vol., I44 p., Masson, Paris.

SEBER (i. A. F., LE CRFN E. D., 1967. Estimating populations parameters from catches large to relative populations. J. Anim. Ecol, 36 (3), 63I-643.

Timmermans J. A., r957. Estimation des populations piscicoles. Application aux eaux courantes rhéophiles. Trav. Sta. Kech. Groenendaal, série D, 21, $84 \mathrm{p}$. 